\title{
Multilayer Neural Network-Based Battery Module SOH Diagnosis
}

\author{
Jong-Hyun Lee', Hyun-Sil Kim² ${ }^{2}$ and In-Soo Lee ${ }^{3}$ \\ ${ }^{1}$ School of Electronics Engineering Kyungpook National University, Korea. \\ ${ }^{2}$ Naval Combat Systems PMO Agency For Defence Development, Korea. \\ ${ }^{3}$ Corresponding author, School of Electronics Engineering Kyungpook National University, Korea.
}

\begin{abstract}
Today, lithium batteries are used in a variety of applications such as cell phones, electric vehicles, unmanned submarines, and energy storage systems (ESS) as the primary power source. Therefore, for stable use, it is important for the device and the system to quickly detect any fault occurring in the battery and accurately diagnose it. Battery faults can be diagnosed from the battery's state of health $(\mathrm{SOH})$ which reflects its operating condition. In this paper, a system is proposed to diagnose battery cell faults by means of a multilayer neural network (MNN) state classifier. In this method, the MNN state classifier utilizes the discharge voltage data obtained by operating the lithium battery cell at high temperature. We concluded from experimental results that the proposed battery $\mathrm{SOH}$ monitoring method diagnoses the state of the battery very well.
\end{abstract}

Keywords: Lithium battery, State of Health, Fault diagnosis system, Multilayer Neural Network

\section{INTRODUCTION}

Lithium batteries are widely used in cell phones, electric vehicles, unmanned submarines, ESSs, and are the main power source in many other systems [1][2]. Battery faults result in a performance reduction in individual devices and can be critical at the system level [3][4]. Therefore, for reliable operation, it is important to promptly detect defects in batteries and accurately determine faults. In general, the status of a battery can be diagnosed by analyzing its $\mathrm{SOH}$. In existing methods, $\mathrm{SOH}$ is roughly measured based on the number of service hours or the number of charging and discharging cycles specified by the battery manufacturer, but a battery's life can be reduced or fault more quickly depending on the temperature and operating conditions.

There are various ways to determine battery SOH. In [5], the resistance of a battery's equivalent circuit and a Kalman-filter method predicted the SOH of the battery. However, the variation in the internal resistance of a battery is very small, so the changes of resistance are not enough to precisely diagnose the $\mathrm{SOH}$. The open-circuit voltage (OCV) method measures voltage under no-load conditions and predicts $\mathrm{SOH}$ according to OCV changes [6]. However, the Kalman-filter method has the disadvantage that it is difficult to apply due to the complexity of its parameters and algorithms.

Neural networks are currently applied to solve problems in areas like pattern recognition, identification, and classification.
Neural networks have three representative features. First, they have the ability to learn complex nonlinear input and output relationships. Second, they use sequential training procedures. Third, they can adapt to data. For these reasons, neural networks have proven to be a promising technology for intelligent machines [7]. They can implement algorithms that learn from data and can execute data-driven decisions or predictions. Neural networks can efficiently model a variety of input and output relationships; when compared to a procedural model, they have the advantage of shorter execution time [8][9]

In this paper, we propose $\mathrm{SOH}$ monitoring of a battery module using an MNN. Lithium battery characteristics and performance are affected by changes in temperature [10][11]. In an experiment to diagnose a battery's state, the battery was operated at a high temperature $\left(40^{\circ} \mathrm{C}\right)$ and a low temperature $\left(-10^{\circ} \mathrm{C}\right)$ for a long time. The battery's fault state was diagnosed by an MNN that was trained to recognize normal, warning, and fault conditions using the battery's charging and discharging data.

\section{THE MONITORING SYSTEM BASED ON MNN}

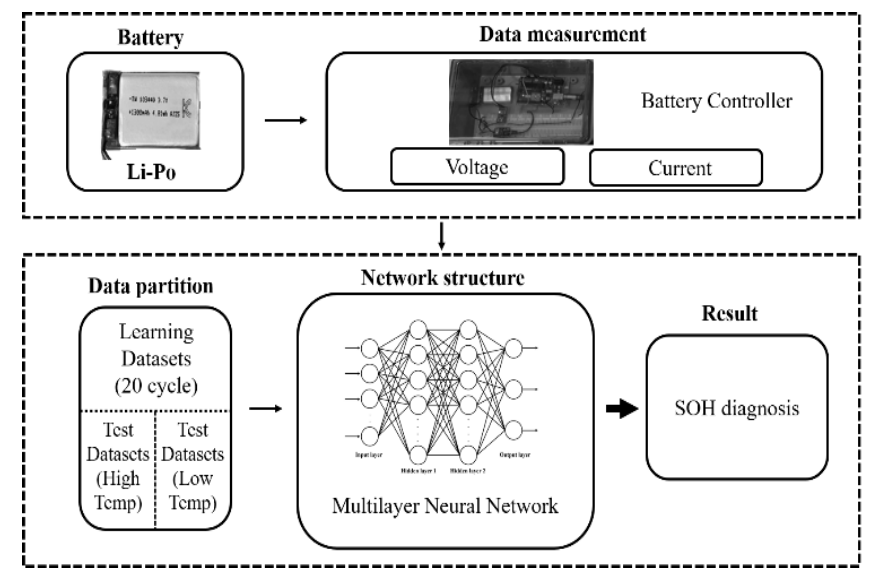

Fig. 1. Configuration of the battery $\mathrm{SOH}$ monitoring system

The proposed lithium battery $\mathrm{SOH}$ monitoring system is shown in Fig. 1. It consists of a battery, a battery controller, and an MNN state classifier. The battery controller comprises a protection circuit and a sensor that measures voltage and current data and sends it to a PC when the battery is charged or 
discharged. The MNN receives data from the battery controller and diagnoses the battery state diagnoses the battery as being in one of three states: normal, warning, or fault.

\section{II.1 Lithium Battery Experiment Set-up}

The energy storage capacity of a battery is defined as the total amount of charge removed when a constant current discharges the battery from a fully charged state to a fully discharged state. The fully charged state is defined as the state in which no current flows by applying a constant voltage. The fully discharged state is defined as draining all charge within a range where the battery is not damaged.

The test method exposes a fully charged battery to high temperature $\left(40^{\circ} \mathrm{C}\right)$ or low temperature $\left(-10^{\circ} \mathrm{C}\right)$ for 8 hours. The battery was then exposed to room temperature for 3 hours and subsequently discharged.

\section{2 Multilayer Neural Network State Classifier}

An MNN is the most basic learning model in deep learning and has two or more hidden layers. It allows a high level of abstraction by combining various nonlinear transform techniques with more hidden layers in existing neural networks [12].

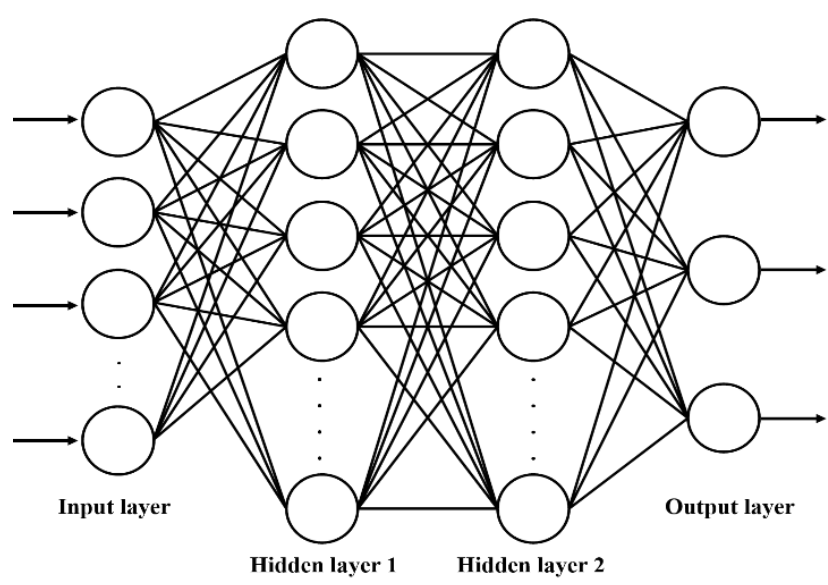

Fig. 2. Structure of the lithium battery charge-discharge experimental MNN

The MNN state classifier used in this paper consists of one input layer, two hidden layers, and one output layer as shown in Fig. 2. The activation function ReLU was used for each hidden layer, and softmax was used for the output layer. The input layer has 3600 nodes; the hidden layers 1 and 2 have 256 nodes. The three nodes in the output layer are for the normal, warning, and fault state outputs. The optimizer function uses the RMSprop function and the loss function uses the crossentropy function.

ReLU is the most commonly used activation function in recent deep learning research [13]. ReLU solves the problem of gradient vanishing in the sigmoid function and has the advantage of faster convergence than the sigmoid function. ReLU is shown in Equation (1).

$$
f(x)=\left\{\begin{array}{c}
x \text { for } x>0 \\
0 \text { for otherwise }
\end{array}\right.
$$

The RMSprop algorithm does not uniformly add all past gradients but reflects only the information of the new gradient so that the learning rate does not become 0 [14]. The RMSprop algorithm is as shown in Equation (2) and Equation (3).

$$
\begin{gathered}
G_{t}=\gamma G+(1-\gamma)(\nabla \mathrm{J}(W(t)))^{2} \\
W(t)=W(t-1)-\sqrt{\frac{\eta}{G_{t}-\epsilon}} \cdot \nabla \mathrm{J}(W(t-1))
\end{gathered}
$$

where $\gamma$ is the forgetting factor, typically 0.9. $\theta$ is a parameter of the network and $f(\theta)$ is a cost function. In this paper, softmax is used. $\nabla_{\theta} f(\theta)$ is the gradient of the network and $t$ is a time step. For the network parameter $\mathrm{k}, \mathrm{G}_{t}$ is a k-dimensional vector that stores the sum of squares of the gradient to which each variable moves until time step t. $\eta$ is the learning rate and is set to $0.001 . \epsilon$ is a small value of about $10^{-4}$ to $10^{-8}$ to prevent division by 0 .

The softmax function is used for the output layer and to transform the classification problem. The output from the previous layer determines the probability of each state when solving the state classification problem. Softmax takes the exponent of each output and divide by the normalization constant so that the sum is 1 . The softmax function is shown in Equation (4).

$$
p_{i}=\frac{\exp \left(x_{i}\right)}{\sum_{k} \exp \left(x_{k}\right)}
$$

where $k$ is the number of dimensions of the input and output vectors. $p_{i}$ is the $i$-th output value. $x_{i}$ is the $i$-th input value.

\section{EXPERIMENT AND RESULTS}

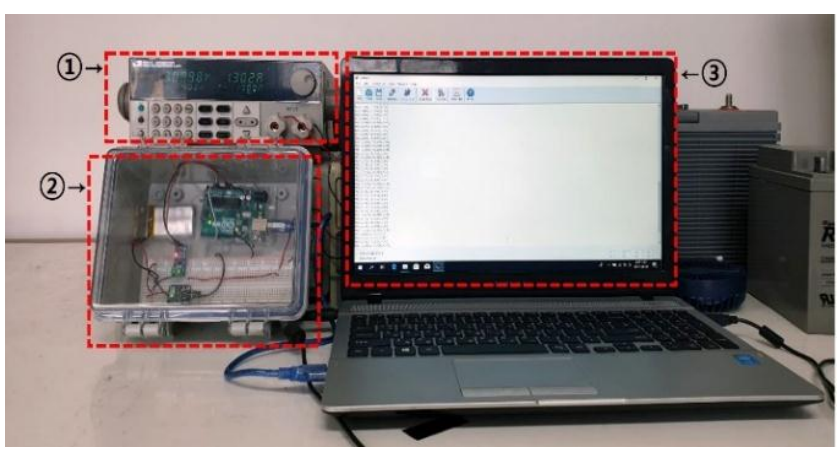

Fig. 3. Circuit configured for the experiment

The experimental setup configuration for conducting the experiment is shown in Fig. 3. Fig. 3-(1) is an electronic load, and when charging, it is replaced with a power supply. Fig. 3(2) is a battery system and consists of a battery, a current and voltage sensor, and an MCU (Micro Controller Unit) to 
communicate with the PC. Fig. 3-(3) is the PC and serial communication with the battery system to receive battery voltage and current data and monitor the battery $\mathrm{SOH}$.

Table 1. Battery cell specification

\begin{tabular}{|c|c|}
\hline Battery type & Li-Po battery \\
\hline Capacity & $1300[\mathrm{mAh}]$ \\
\hline Voltage Range & $2.4 \sim 4.28[\mathrm{~V}]$ \\
\hline Nominal Voltage & $3.7[\mathrm{~V}]$ \\
\hline
\end{tabular}

In order to evaluate the performance of the proposed system, the discharge data of the lithium battery tested in the high temperature and low-temperature environments as shown in Fig. 4 were used for learning. According to Yinjiao Xing's paper, a battery should be replaced when its capacity is reduced to $80 \%$ of its rated beginning of life capacity [15]. In the experiment of this paper, we defined $100 \%$ to $90 \%$ of the rated capacity as normal, 90 to $80 \%$ of the rated capacity as a warning, and less than $80 \%$ of the rated capacity as a fault. In Fig. 4(a), patterns 1 to 5 were learned as normal, patterns 6 and 7 as warnings, and patterns 8 to 10 as faults. In Fig. 4(b) patterns 1 and 2 were learned as normal, patterns 3 to 7 as warnings, and patterns 8 to 10 as faults.

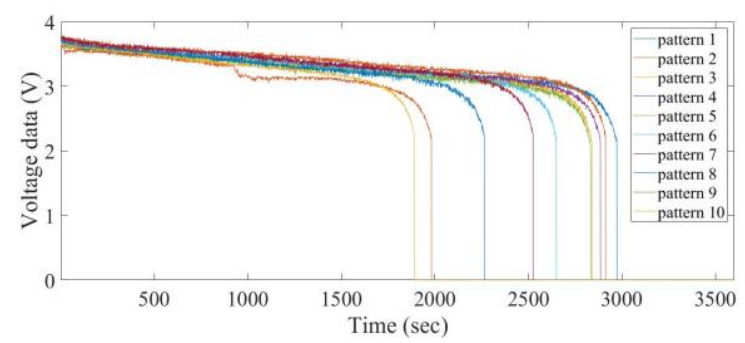

(a) Battery discharge voltage data used in learning at high temperature

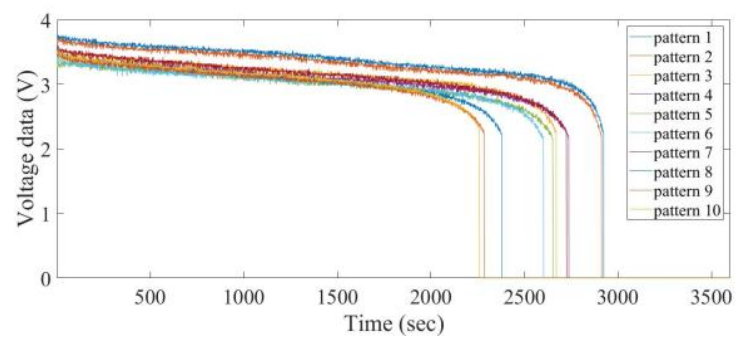

(b) Battery discharge voltage data used in learning at low temperature

Fig. 4. Lithium battery discharge voltage data for learning

Fig. 5 is the discharge graph of another battery of the same type operated in the high-temperature environment of the test. In Fig. 5(a), patterns 1 and 2 are normal, patterns 3 to 5 are warnings, and patterns 6 to 8 are faults. The MNN's SOH diagnostic test result for the battery operated at high temperature is shown in Fig. 5(b).

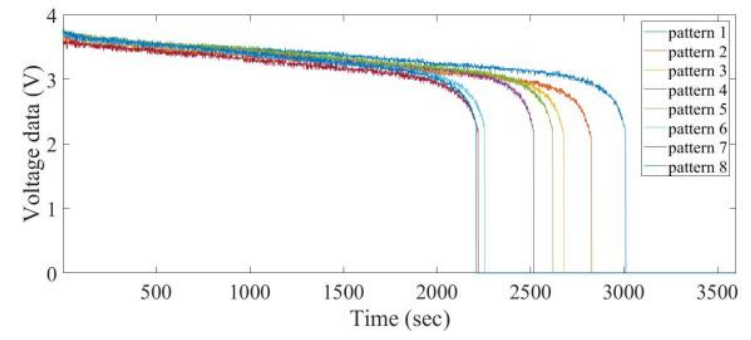

(a) Battery discharge voltage data used in test at high temperature

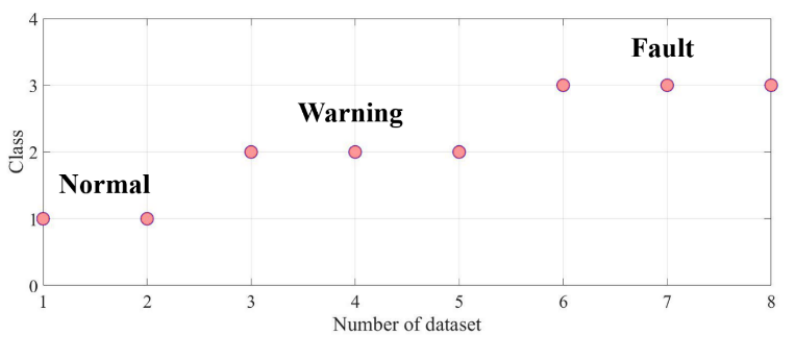

(b) MNN SOH diagnosis test result from a battery operated at high temperature

Fig. 5. Lithium battery test result at high temperature

Fig. 6 shows test data obtained in the low-temperature experimental environment. In Fig. 6(a), patterns 1 and 2 are normal, pattern 3 is a warning, and patterns 4 and 5 are faults. The MNN's SOH diagnostic test result for the battery operated at low temperature is shown in Fig. 6(b).

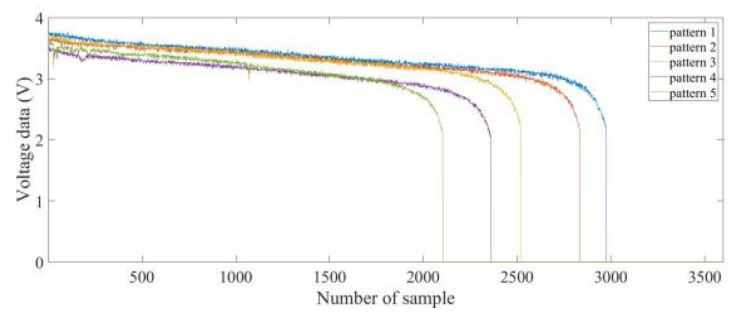

(a) Battery discharge voltage data used in test at low temperature

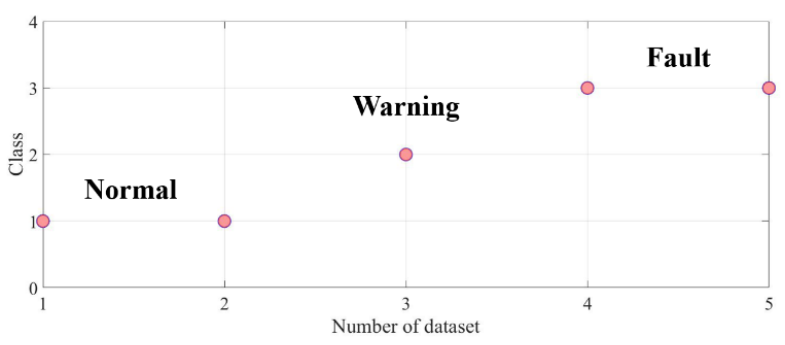

(b) $\mathrm{SOH}$ diagnosis test result of battery operated at low temperature by MNN

Fig. 6. Lithium battery test result at low temperature 


\section{CONCLUSION}

In this paper, a lithium battery $\mathrm{SOH}$ monitoring system using an MNN was described. The battery cell was charged and discharged at a high temperature $\left(40^{\circ} \mathrm{C}\right)$ and at a low temperature $\left(-10^{\circ} \mathrm{C}\right)$, and the fault state of the battery was diagnosed by the proposed MNN using the discharge data of the battery.

The experimental results show that the proposed state classifier diagnoses the actual state of the battery well; this was verified by testing with the discharge data of a lithium battery of the same specification as the one used to teach the MNN and operating it under the same experimental conditions. In future work, we plan to apply the proposed MNN-based method to multiple battery cells.

\section{ACKNOWLEDGMENT}

This research was supported by Basic Science Research Program through the National Research Foundation of Korea (NRF) funded by the Ministry of Education (No. 2019R1I1A3A01058319).

This study was supported by the BK21 Plus project funded by the Ministry of Education, Korea (21A20131600011).

\section{REFERENCES}

[1] Hwang H R, Kim B S, Xui R, Lee I S. Development of A Fault Diagnosis Algorithm for Solar Panel. Proceedings of IEIE Summer Conference. 2016;14631465.

[2] Cho, T H, Hwang H R, Lee J H, Lee I S. Comparison of Intelligent Methods of SOC Estimation for Battery of Photovoltaic System. International Journal of Advanced Computer Science and Applications. 2018;9(9):49-56.

[3] Sidhu A, Izadian A, Anwar S. Adaptive nonlinear model-based fault diagnosis of Li-ion batteries. IEEE Transactions on Industrial Electronics. 2014;62(2):1002-1011.

[4] Sidhu A, Izadian A, Anwar S. Fault diagnosis of Li-Ion batteries using multiple-model adaptive estimation. In IECON 2013-39th Annual Conference of the IEEE Industrial Electronics Society. 2013;3524-3529.

[5] Andre D, Appel C, Soczka-Guth T, Sauer D U. Advanced mathematical methods of SOC and $\mathrm{SOH}$ estimation for lithium-ion batteries. Journal of power sources. 2013;224:20-27.

[6] $\mathrm{Ng} \mathrm{K} \mathrm{S,} \mathrm{Moo} \mathrm{C} \mathrm{S,} \mathrm{Chen} \mathrm{Y} \mathrm{P,} \mathrm{Hsieh} \mathrm{Y} \mathrm{C.} \mathrm{Enhanced}$ coulomb counting method for estimating state-of-charge and state-of-health of lithium-ion batteries. Applied energy. 2009;86(9):1506-1511.

[7] Schürmann J. Pattern classification: a unified view of statistical and neural approaches. John Wiley \& Sons, Inc. 1996.
[8] Fausett L. Fundamentals of neural networks: architectures, algorithms, and applications. PrenticeHall, Inc. 1994.

[9] Elshatter T F, Elhagry M T, Abou-Elzahab E M, Elkousy A A T. Fuzzy modeling of photovoltaic panel equivalent circuit. In Conference Record of the TwentyEighth IEEE Photovoltaic Specialists Conference-2000 (Cat. No. 00CH37036). 2000;15(22):1656-1659.

[10] Wright R B, et al. Power fade and capacity fade resulting from cycle-life testing of advanced technology development program lithium-ion batteries. Journal of Power Sources. 2003;119:865-869.

[11] Burke A, Miller M. Life cycle testing of lithium batteries for fast charging and second-use applications. In 2013 World Electric Vehicle Symposium and Exhibition (EVS27). 2013;1-10.

[12] Hinton G E, Osindero S, Teh Y W. A fast learning algorithm for deep belief nets. Neural computation. 2006;18(7):1527-1554.

[13] Clevert D A, Unterthiner T, Hochreiter S. Fast and accurate deep network learning by exponential linear units (elus). arXiv preprint arXiv:1511.07289 [Internet]. 2015.

[14] Tieleman T, Hinton G. Lecture 6.5-rmsprop: Divide the gradient by a running average of its recent magnitude. COURSERA: Neural networks for machine learning. 2012;4(2):26-31.

[15] Xing Y, Ma E W, Tsui K L, Pecht M. Battery management systems in electric and hybrid vehicles. Energies. 2011;4(11):1840-1857. 